\title{
The Bloom's Syndrome Helicase Interacts Directly with the Human DNA Mismatch Repair Protein hMSH6
}

\author{
Graziella Pedrazzi' ${ }^{1}$, Csanád Z. Bachrati², \\ Nives Selak'1, Ingrid Studer ${ }^{1}$, Maja Petkovic', \\ Ian D. Hickson ${ }^{2}$, Josef Jiricny ${ }^{3}$ and \\ Igor Stagljar ${ }^{1, *}$ \\ ${ }^{1}$ Institute of Veterinary Biochemistry and Molecular \\ Biology, University of Zürich, Winterthurerstr. 190, \\ $\mathrm{CH}-8057$ Zürich, Switzerland \\ 2 Cancer Research UK Laboratories, Weatherall Institute \\ of Molecular Medicine, University of Oxford, John \\ Radcliffe Hospital, Oxford OX3 9DS, UK \\ ${ }^{3}$ Institute of Molecular Cancer Research, University of \\ Zürich, August Forel-Strasse 7, CH-8008 Zürich, \\ Switzerland
}

${ }^{*}$ Corresponding author

Bloom's syndrome (BS) is a rare genetic disorder characterised by genome instability and cancer susceptibility. BLM, the BS gene product, belongs to the highly-conserved RecQ family of DNA helicases. Although the exact function of BLM in human cells remains to be defined, it seems likely that BLM eliminates some form of homologous recombination (HR) intermediate that arises during DNA replication. Similarly, the mismatch repair (MMR) system also plays a crucial role in the maintenance of genomic stability, by correcting DNA errors generated during DNA replication. Recent evidence implicates components of the MMR system also in HR repair. We now show that hMSH6, a component of the heterodimeric mismatch recognition complex hMSH2/hMSH6 (hMutS $\alpha$ ), interacts with the BLM protein both in vivo and in vitro. In agreement with these findings, BLM and hMSH6 colocalise to discrete nuclear foci following exposure of the cells to ionising radiation. However, the purified recombinant MutS $\alpha$ complex does not affect the helicase activity of BLM in vitro. As BLM has previously been shown to interact with the hMLH1 component of the hMLH1/hPMS2 (hMutL $\alpha$ ) heterodimeric MMR complex, our present findings further strengthen the link between BLM and processes involving correction of DNA mismatches, such as in the regulation of the fidelity of homologous recombination events. Key words: BLM helicase/Bloom's syndrome (BS)/ Genome stability/Homologous recombination (HR)/ Mismatch repair (MMR)/Protein-protein interactions.

\section{Introduction}

Bloom's syndrome (BS) is a rare autosomal recessive disorder characterised by growth retardation, immunodeficiency, sun sensitivity, genomic instability, and a strong predisposition to many types of cancer (German, 1993). $\mathrm{BS}$ is caused by mutations in the BLM gene, which encodes a DNA helicase of the RecQ family (Ellis et al., 1995). Other members of this highly-conserved group of proteins include the E. coli RecQ (Nakayama et al., 1985), S. cerevisiae Sgs1p (Gangloff et al., 1994), S. pombe Rqh1p (Stewart et al., 1997), and four additional human homologues, RECQL (Puranam and Blackshear, 1994); $W R N$, the gene mutated in the premature aging disorder Werner's syndrome (Yu et al., 1996); RECQL4, the gene mutated in Rothmund-Thomson syndrome (Kitao et al., 1999); and RECQL5 (Kitao et al., 1998). The most obvious abnormalities observed in cells derived from BS individuals are the highly increased frequency of sister-chromatid exchanges (SCEs), chromatid breaks and gaps, and rearranged chromosomes (German et al., 1974). The BLM gene encodes a nuclear protein consisting of 1417 amino acids (Ellis et al., 1995) that possesses a 3' $\rightarrow$ 5' DNA helicase activity on a variety of different DNA substrates (Karow et al., 1997; Sun et al., 1998; Mohaghegh et al., 2001). The BLM mutations in BS individuals disrupt the helicase activity of BLM, often resulting in the expression of an mRNA that is unstable. This suggests that BS results from the absence of BLM helicase activity/protein, and that BLM is not an essential protein in humans (Ellis et al., 1995). Consistent with this hypothesis, a viable mouse model of BS has been developed through gene targeting, in which BLM mRNA and protein expression are absent or undetectable (Luo et al., 2000). Immunofluorescence studies revealed that BLM is present in nuclear bodies containing the promyelocytic leukemia protein (PML) (Ishov et al., 1999; Sanz et al., 2000; Yankiwski et al., 2000; Bischof et al., 2001), in diffuse patches in the nucleolus (Yankiwski et al., 2000), and at telomeres in telomerase-negative tumor cells (Stavropoulos et al., 2002).

Although the precise function of BLM in human cells remains poorly defined, considerable evidence suggests that the BLM protein plays a role in homologous recombination (HR), probably by participating in the process of HR-mediated replication restart at sites of stalled replication forks. Genetic studies utilising the S. cerevisiae SGS1 gene have played a crucial role in furthering our understanding of the function(s) of RecQ helicases such as BLM in the process of HR. Deletion of SGS1 leads to an increase in the frequency of several types of DNA recom- 
bination, as well as a breakdown in the fidelity of chromosome segregation during mitosis and meiosis (Gangloff et al., 1994; Watt et al., 1995, 1996). In addition, interspecies cross-functionality between BLM and $S G S 1$ has been indicated by the observation that $B L M$ is capable of partially suppressing the hyper-recombination phenotype of sgs1 mutants and restoring the slow growth phenotype of a top3 sgs1 double mutant (Yamagata et al., 1998). Further evidence for a role of BLM in HR comes from the observation that BLM directly interacts with RAD51, and co-localises with it in the nucleus of cells exposed to DNA damaging agents (Bischof et al., 2001; Wu et al., 2001). Furthermore, the enzymatic activity of the BLM helicase is also consistent with a role in HR: BLM can disrupt synthetic D-loop structures and catalyse branch migration of Holliday junctions $(\mathrm{HJ})$ and synthetic four-way junctions (Karow et al., 2000; van Brabant et al., 2000; Mohaghegh et al., 2001), which arise as intermediates during $\mathrm{HR}$, and may occur spontaneously during DNA replication and repair (Karow et al., 2000). BLM has recently been shown to interact with p53 (Garkavtsev et al., 2001; Wang et al., 2001). This interaction attenuates the ability of BLM to unwind synthetic $\mathrm{HJ}$ in vitro (Yang et al., 2002) and is necessary to transport p53 to sites of stalled DNA replication forks where the two proteins functionally interact to modulate HR (Sengupta et al., 2003). Moreover, the presence of BLM in the two multiprotein complexes BASC (BRRCA1-Asssociated genome Surveillance Complex), together with hMSH2, hMSH6, hMLH1, ATM, the RAD50-MRE11-NBS1 complex and replication factor C (Wang et al., 2000), and BRAFT, containing also five Fanconi anemia complementation group proteins, topoisomerase III $\alpha$ and replication protein A (Meetei et al., 2003), suggests a functional link to DNA repair.

The DNA mismatch repair (MMR) system is a conserved pathway involved in the removal of mispaired bases from DNA, which plays an important role in the maintenance of genomic stability in both prokaryotes and eukaryotes (reviewed in Bellacosa, 2001). The E. coli MutHLS MMR pathway has been well characterised biochemically and genetically (Modrich and Lahue, 1996), and has served as a paradigm for the yeast and mammalian MMR pathways. A number of homologues of MutS and MutL MMR proteins have been described in yeast and mammalian cells. Base/base mismatches and small insertion/deletion loops are recognised by the hMutS $\alpha$ complex, which is a heterodimer of hMSH2 and hMSH6. hMSH2 also pairs with another MutS homologue, hMSH3, to form a heterodimer known as hMutSB, which is involved in the repair of larger insertion/deletion loops. Following this initial mismatch-recognition step, homologues of the bacterial MutL ATPase, predominantly the hMLH1-hPMS2 (hMutL $\alpha$ ) heterodimer in humans, couple mismatch recognition to the appropriate downstream processing steps. Interestingly, defects in some of the MMR proteins lead to an inherited cancer syndrome called hereditary non-polyposis colon cancer
(HNPCC). Mutations in two MMR genes, hMSH2 and $h M L H 1$, have typically been associated with HNPCC, while mutations in other MMR genes (hMSH6, hPMS1, and $h P M S 2$ ) are rare.

In addition to their role in the repair of replication errors, MMR proteins have been implicated in some aspects of HR (reviewed in Evans and Alani, 2000; Bellacosa, 2001). In mammalian cells, as in other organisms, $\mathrm{HR}$ is well established as one of the major pathways for the repair of DNA double-strand breaks. MMR components have been shown to function in HR by suppressing recombination between homeologous sequences (similar, but not identical), a role that appears to be conserved in bacteria, yeast, and mammals (Modrich and Lahue, 1996). The hMutS $\alpha$ complex has also been shown to bind to HJs, suggesting that it may be involved in additional HR processes in vivo (Marsischky et al., 1999). Moreover, $\mathrm{hMSH}^{-/-}$and $\mathrm{hMSH}^{-/-}$murine embyonic stem cells are promiscuous during recombination between homologous sequences in gene-targeting experiments (de Wind et al., 1995, 1999).

We and others have recently demonstrated that BLM interacts directly with the MMR protein $\mathrm{hMLH} 1$ (Langland et al., 2001; Pedrazzi et al., 2001). Since BS cells are not deficient in MMR, it has been proposed that the hMLH1 interaction with BLM may play a role in HR (Langland et al., 2001; Pedrazzi et al., 2001). In this study, we set out to test whether BLM interacts also with other components of the MMR system. We demonstrate here that BLM interacts directly with hMSH6, but not with the $\mathrm{hMSH} 2$ component of the MutS $\alpha$ heterodimer. Consistent with this notion is the observation that BLM and hMSH6 co-immunoprecipitate from human nuclear extracts and co-localise to nuclear foci in response to ionising radiation. Taken together, our data provide further evidence for a role of BLM helicase alongside MMR proteins in HR.

\section{Results}

\section{BLM Directly Interacts with hMSH6 But Not with hMSH2}

To examine whether BLM interacts with components of the MMR system in addition to hMLH1, we performed a dot-blot assay where we immobilised increasing amounts of purified recombinant MutS $\alpha$ (laccarino et al., 1998), MutL $\alpha$ (positive control) (Raschle et al., 1999), or phage protein $D$ (negative control), onto a nitrocellulose membrane, and incubated the membrane with purified recombinant BLM protein (Karow et al., 1997). The presence of bound BLM protein was detected using an antibody against BLM. As shown in Figure 1A, BLM interacts with the MutS $\alpha$ heterodimer, although to a slightly lesser extent than with the MutL $\alpha$ complex. Knowing that BLM directly interacts with at least one of the components of MutS $\alpha$ complex, we sought to identify whether this inter- 


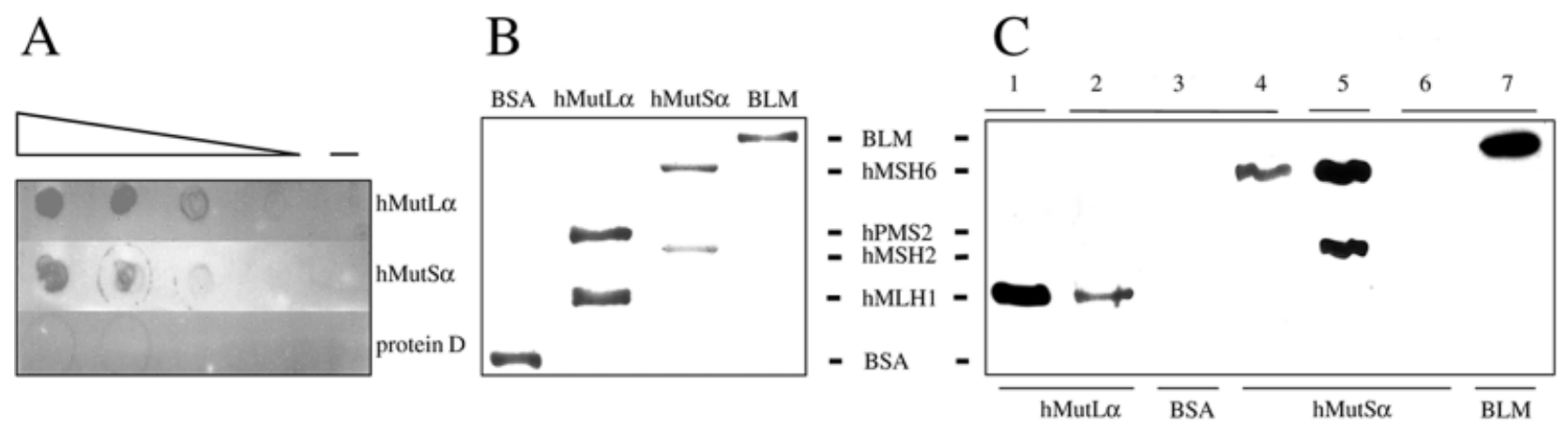

Fig. 1 BLM Interacts Directly with hMSH6, But Not with hMSH2.

(A) Purified recombinant BLM can bind to immobilised purified recombinant MutS $\alpha$. The bound BLM was detected using an anti-BLM antibody. (B) Coomassie Blue stained gel showing the purified BSA (1 $\mu \mathrm{g})$, MutL $\alpha(1.5 \mu \mathrm{g})$, MutS $\alpha(1 \mu \mathrm{g})$ and BLM proteins (1 $\mu \mathrm{g})$ used in (C). (C) Far-Western analysis. $0.5 \mu \mathrm{g}$ of MutL $\alpha$ (lanes 1, 2), $1 \mu \mathrm{g}$ of BSA (lane 3), $1 \mu \mathrm{g}$ of MutS $\alpha$ (lanes $4-6$ ) and $0.2 \mu \mathrm{g}$ of BLM (lane 7 ) were separated by SDS-PAGE and blotted onto a nitrocellulose membrane. After renaturation, lanes 2 to 4 of the membrane were incubated with purified recombinant BLM $(1 \mu \mathrm{g} / \mathrm{ml})$ and the presence of bound BLM protein was detected by Western analysis using an anti-BLM antibody. Lane 1 was probed with an antibody against hMLH1, lane 5 with antibodies against hMSH2 and hMSH 6 and lanes 6 and 7 were probed with an anti-BLM antibody.

A

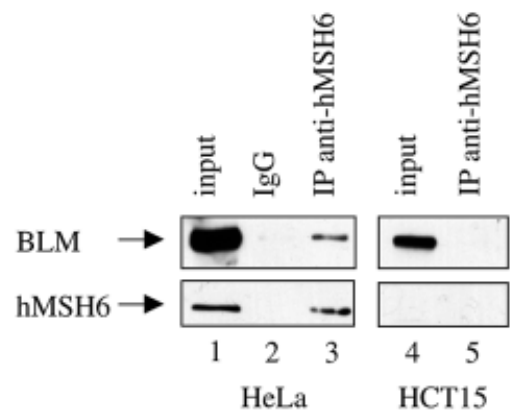

B

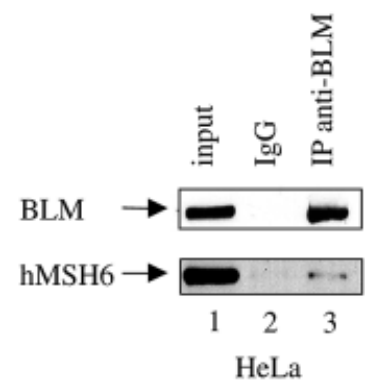

Fig. 2 BLM and hMSH6 Exist as a Complex in Human Cells.

(A) Co-immunoprecipitation of BLM with hMSH6. BLM could be co-immunoprecipitated with an anti-hMSH6 antibody from $200 \mu \mathrm{g}$ of HeLa nuclear extract (lane 3), but not with IgG (lane 2). Lane 1 shows the input (20 $\mu \mathrm{g})$. The proteins were visualised by Western blot analysis with antibodies against BLM (upper) or hMSH6 (lower). (B) Co-immunoprecipitation of hMSH6 with BLM. hMSH6 was immunoprecipitated from $200 \mu \mathrm{g}$ of HeLa nuclear extract (lane 3) with an anti-BLM antibody but not with IgG (lane 2). The proteins were detected with antibodies against BLM (upper) or hMSH6 (lower).

action was with hMSH2 and/or hMSH6. For this, we performed a Far-Western blot analysis. Figure 1B shows a Coomassie Blue stained gel with the purified recombinant proteins. MutS $\alpha$ was separated into its two components, hMSH2 and hMSH6, by SDS-PAGE and the proteins were then renatured on the membrane after blotting. Subsequently, lanes 2 to 4 (Figure 1C) of the membrane were probed with purified recombinant BLM protein. Western analysis with an anti-BLM antibody (IHIC33) revealed bands at the positions corresponding to hMHS6 (lane 4) and the positive control hMLH1 (lane 2) after incubation with BLM as well as for the BLM input (lane 7). No signal was detected at the positions of the negative controls hPMS2 (lane 2) and BSA (lane3) nor at the position of the hMSH2 protein (lane4). Cross-reactivity of the anti BLM antibody with hMSH6 can be excluded, as it did not recognize the protein that had not been incubated with BLM (lane 6). The amount of BLM bound to hMSH6 strongly increased when MutL $\alpha$ complex was added to the reaction (data not shown), although the mechanism underlying this effect is not known and awaits further investigation.

\section{BLM and hMHS6 Form a Complex in Human Cells}

To confirm the BLM/hMSH6 interaction detected by the Far-Western assay, and to assess whether this interaction can be detected in human cells, we performed coimmunoprecipitation experiments on nuclear extracts from HeLa cells. Using a monoclonal antibody against hMSH6, we were able to specifically co-immunoprecipitate BLM (Figure 2A, lane 3). No BLM was present in the precipitate when a control antibody was used (Figure 2A, lane 2). Similarly, BLM could not be co-immunoprecipitated with an anti-hMSH6 antibody from hMSH6-deficient HCT15 nuclear extracts (Figure 2A, lane 5). Reciprocal co-immunoprecipitation experiments showed that hMSH6 could specifically be precipitated with a poly- 
clonal anti-BLM antibody (Figure 2B, lane 3) from HeLa nuclear extracts. We observed no increase in the amount of co-immunoprecipitated BLM/hMSH6 complex upon the addition of native DNA or mismatched DNA (data not shown).

\section{Mapping of BLM and hMSH6 Interaction Regions}

To investigate the region of BLM protein that is responsible for mediating the interaction with hMSH6, different BLM deletion mutants were transcribed and translated in vitro (IVTT) and used as radioactive probes to test for their ability to interact with full-length $\mathrm{hMSH} 6$ in an in vitro binding assay (IBA). As described above, the recombinant MutS $\alpha$ complex was separated by SDS-PAGE and its constituent polypetides were renatured on the membrane after blotting. As a negative control, BSA was included on the membrane. Where possible, based on previous mapping data, the translated BLM fragments were tested for their binding to hMLH1, in order to confirm the correct folding of the IVTT BLM fragments. As shown in Figure $3 \mathrm{~A}$, the result of the IBA revealed two separate regions on BLM that are required for binding to $\mathrm{hMSH} 6$. While no interaction could be observed with the N-terminal BLM-construct (amino acids 1 -448), both an internal fragment covering amino acids 340-770, and a C-terminal fragment spanning amino acids 904-1417 showed binding to hMSH6. Neither of these fragments bound to BSA or hMSH2. We conclude that BLM contains two separate hMSH6-binding regions.

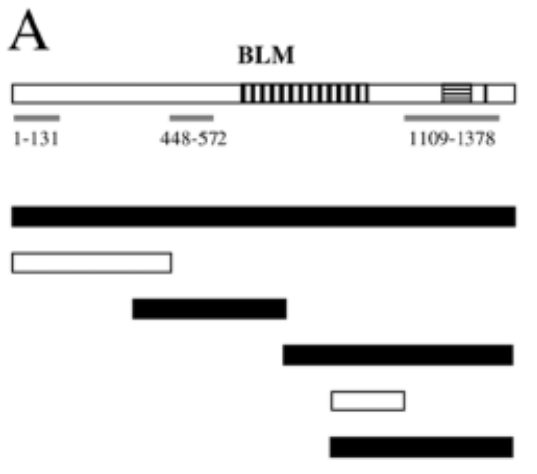

$1-1417$

$1-1417$

$1-448$

340-770

770-1417

904-1108

904-1417

IBA

\begin{tabular}{|c|c|c|c|}
\hline hMSH6 & hMLH1 & BSA & hMSH2 \\
\hline+ & + & - & - \\
- & + & - & - \\
+ & + & - & - \\
+ & + & - & - \\
- & nd & - & - \\
+ & + & - & - \\
\hline
\end{tabular}

B

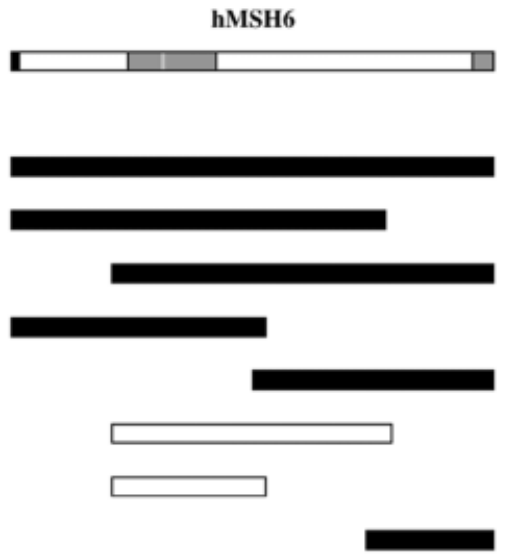

$1-1360$

\begin{tabular}{|c|c|c|}
\multicolumn{4}{c}{ IBA } \\
\hline BLM & MutS $\alpha$ & BSA \\
\hline+ & + & - \\
+ & + & - \\
+ & + & - \\
+ & + & - \\
+ & + & - \\
- & + & - \\
- & + & - \\
+ & + & - \\
\hline
\end{tabular}

Fig. 3 Mapping of BLM- and hMSH6-Interacting Regions by an in vitro Binding Assay.

(A) Two separate regions on BLM interact with hMSH6. Recombinant MutS $\alpha$ complex $(1 \mu \mathrm{g})$, MutL $\alpha(1 \mu \mathrm{g})$ and BSA (1 $\mu \mathrm{g})$ were separated by SDS-PAGE and blotted onto a nitrocellulose membrane. After renaturation, the membranes were incubated with [ ${ }^{55}$ S]-labelled IVTT BLM fragments. Black bars show fragments that were able to bind to hMSH6, but not to hMSH2 or BSA, white bars represent fragments that do not bind to hMSH6. The Table summarises the obtained results with all tested proteins (+: interaction; -: no interaction; nd: not determined). The sketch of BLM shows the position of the helicase domain (vertically striped, amino acids $649-1005)$, the HRDC domain (horizontally striped, amino acids 1212 -1292) and the nuclear localisation signals (black bar, amino acids 1334 - 1349). The grey bars below depict the regions mediating the interaction with hMLH1. (B) hMSH6 interacts with BLM via two distinct regions. Aliquots of $0,0.25$ and $0.5 \mu \mathrm{g}$ of purified recombinant BLM protein or MutS $\alpha$ complex (positive control), or $0,0.5$ and $1 \mu \mathrm{g}$ of BSA (negative control) were spotted onto a nitrocellulose membrane and probed with the indicated IVTT fragments. Black bars represent positive interactions, white bars non-interacting fragments. An overview of all tested interactions is shown in the Table (+: interaction, -: no interaction). The sketch shows the regions of hMSH6 interacting with PCNA (black, amino acids 3-22) and hMSH2 (dark grey, amino acids $326-575$ and $1302-1360$ ) as well as the residue important for mismatch binding (light grey, amino acid 432).

${ }^{*}$ As full-length IVTT hMSH6 was highly prone to degradation, it was co-produced with hMSH2. 
DNA
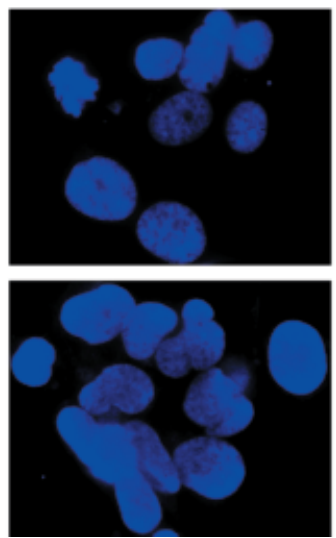

MSH6
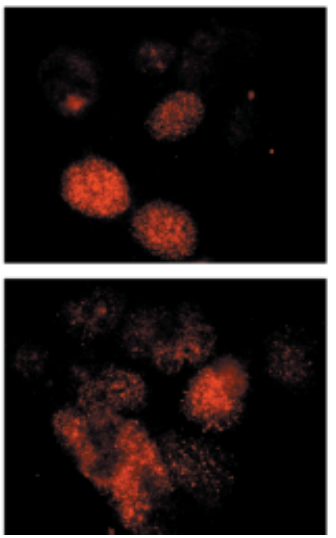

BLM
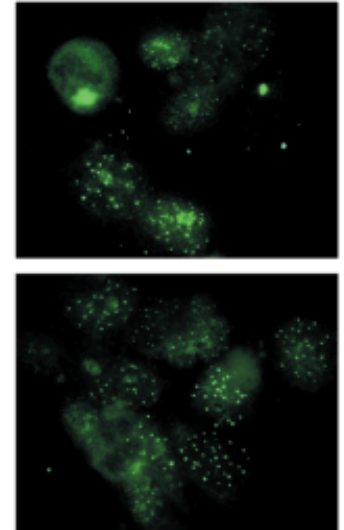

\section{Merge}
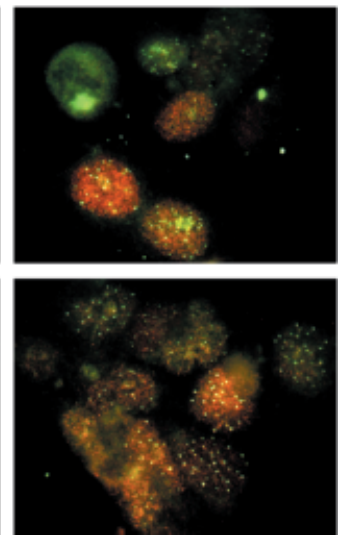

Fig. 4 BLM Co-Localises with hMSH6 to Nuclear Foci in Irradiated WI-38/VA-13 Cells.

Nuclear foci were detected with the rabbit polyclonal IHIC33 anti-BLM antibody (green) and the mouse monoclonal anti-MSH6 (red) antibody in untreated and irradiated WI-38/NA-13 cells as indicated on the left. The merged image indicates where the red and green foci are coincident, as shown by a yellow colour. Nuclear DNA was stained with Hoechst 33258 dye.

To identify which regions of hMSH6 are involved in binding to BLM, we used a similar approach, in which increasing amounts of purified recombinant BLM protein (and MutS $\alpha$ as a positive control and BSA as a negative control) were dotted onto a nitrocellulose membrane, and probed with [ $\left.{ }^{35} \mathrm{~S}\right]$-labelled IVTT deletion mutants of hMSH6, or IVTT full-length hMSH6. Due to rapid degradation when produced alone, hMSH6 was co-translated with $\mathrm{hMSH}$ 2. hMSH2 alone did not interact with BLM (data not shown). Fragments represented by black bars show positive interactions, while white bars show constructs not interacting with BLM (Figure 3B). We conclude therefore, that two BLM-interacting regions are present on $\mathrm{hMSH} 6$, one in the $\mathrm{N}$-terminal half (amino acids $1-718$ ) and one at the very C-terminus (amino acids $1000-1360$ ). A more detailed mapping of the $\mathrm{N}$ terminal fragment was not successful as a fragment covering amino acids 1-305 was not able to bind to a positive control (hMSH6-interacting protein), PCNA, possibly due to misfolding, and therefore we were unable to draw any conclusions about a potential interaction with BLM.

\section{BLM and hMSH6 Co-Localise to Discrete Nuclear Foci in Response to lonising Radiation}

The co-immunoprecipitation of BLM and hMSH6 from human nuclear extracts, as well as the evidence for a direct interaction between purified BLM and hMSH6, is consistent with these proteins forming a complex in vivo and in vitro. To provide additional evidence for this, we asked whether BLM and hMSH6 co-localise within the nucleus of human cells. Previous studies in HeLa and WI38/VA-13 human cells have shown that BLM localises to nuclear foci corresponding to PML nuclear bodies (Ishov et al., 1999; Sanz et al., 2000; Yankiwski et al., 2000; Bischof et al., 2001; Wu et al., 2001), as well as to the nucleolus during certain stages of the cell cycle (Yankiwski et al., 2000). However, BLM has also been shown to colocalise with sites of ongoing DNA replication, at least in a subset of late S-phase cells (Wu et al., 2000a). Immunofluorescence studies conducted with MMR-proficient HeLa cells showed that, in cells undergoing DNA replication, hMSH6 co-localises with PCNA to nuclear foci, which most likely represent replication complexes (Kleczkowska et al., 2001). In untreated WI38/VA-13 cells, we found only very rare cases where BLM-containing nuclear foci appeared to co-localise with hMSH6 (Figure 4, upper panel). We therefore asked if the number of co-localising BLM/hMSH6 foci might increase in response to DNA damage. One hour after 10 Gy of $\gamma$-irradiation, the proportion of cells containing BLM and hMSH6 co-localising foci increased, although co-localisation (at least to discrete foci) was still a rare event (Figure 4, lower panel).

\section{The MutS $\alpha$ Complex Does Not Appear to Influence the DNA Helicase Activity of BLM}

The observation that BLM and $\mathrm{hMSH} 6$ interact directly and can co-localise in nuclear foci following exposure of cells to ionising radiation indicate that the two proteins may be involved in a common cellular pathway. Since the helicase activity of BLM is necessary for the promotion of Holliday junction $(\mathrm{HJ})$ branch migration (Karow et al., 2000; Yang et al., 2002), we investigated whether purified recombinant MutS $\alpha$ complex might modulate the ability of BLM to disrupt a radiolabeled synthetic $\mathrm{X}$-junction substrate, a mimic of the HJ (Figure 5A). Consistent with previous reports (Karow et al., 2000; Yang et al., 2002), the purified recombinant BLM disrupted the $\mathrm{X}$-junction in a dose-dependent manner into primarily two-armed products (the product of branch migration) as well as some one-armed (single-stranded DNA) product generated by the unwinding of the 2-armed species (Figure 5A, 
lanes 3-9). In the absence of BLM, purified recombinant MutS $\alpha$ complex did not show any intrinsic helicase activity (Figure 5A, lane 18). To test the effect of MutS $\alpha$ on the ability of BLM to disrupt the $\mathrm{X}$-junction, increasing amounts of BLM $(8-25 \mathrm{fmol})$ were incubated with the Xjunction in the presence of a molar excess of purified recombinant MutS $\alpha$ complex (200 fmol). No effect of Mut$\mathrm{S} \alpha$ on the DNA helicase activity of BLM was observed

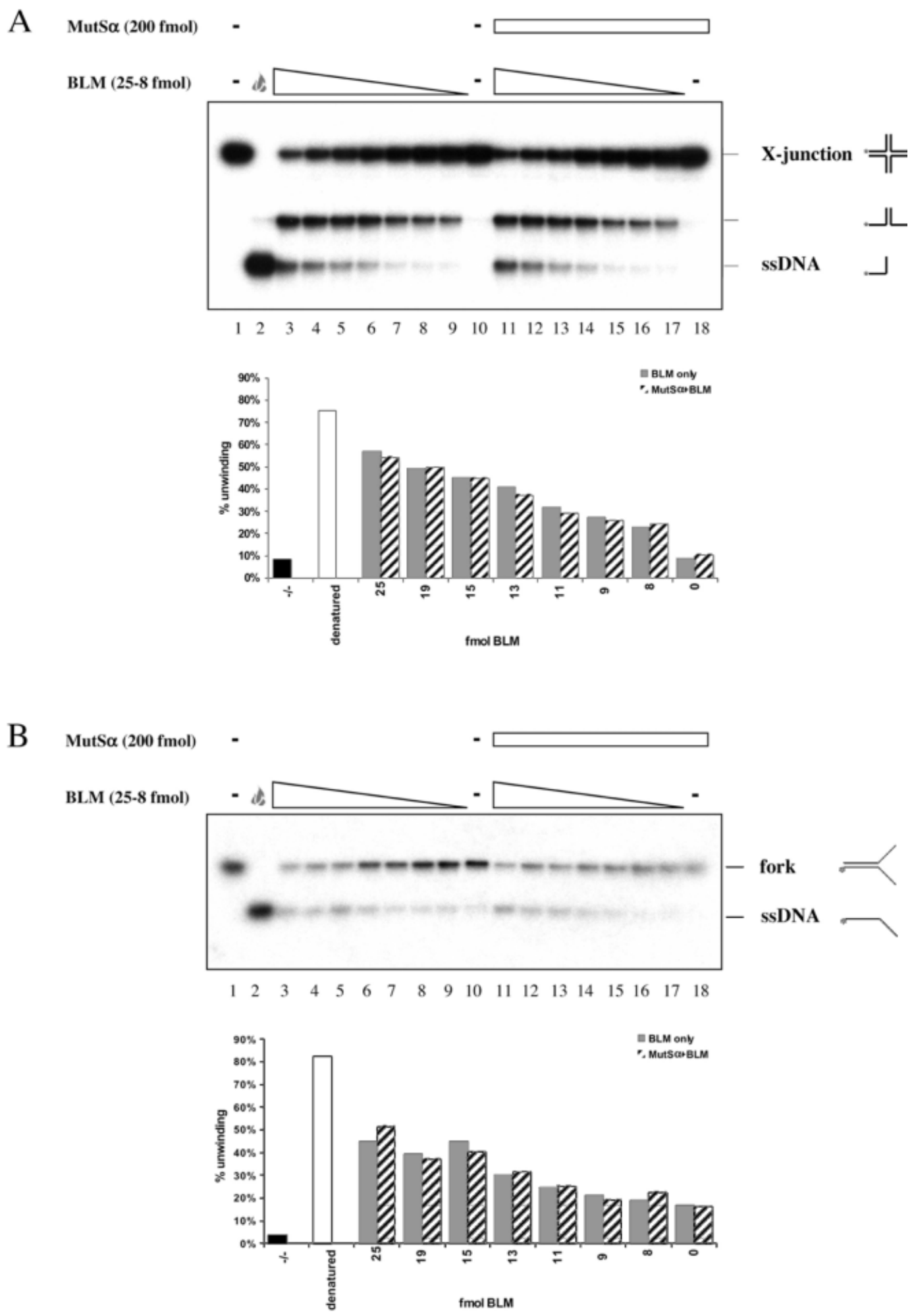

Fig. 5 Helicase Activity of BLM Is Not Affected by the Purified Recombinant MutS $\alpha$ Complex.

Approximately $0.075 \mathrm{fmol}$ of synthetic X-junction (A) or replication fork (B) substrate were incubated with different concentrations (indicated on the chart) of BLM alone or BLM together with $200 \mathrm{fmol}$ MutS $\alpha$. The structures of the intact substrate and products of the unwinding reaction are schematically represented on the right of the autoradiograms. The chart shows quantification of the data from the autoradiogram. The flame symbol depicts heat-denatured substrate (empty bar on chart). 
under these conditions (Figure 5A, chart), or when increasing amounts of purified recombinant MutS $\alpha$ complex $(6.25-400 \mathrm{fmol})$ were used in reactions where the amount of BLM was kept at a fixed level (11.25 fmol; data not shown).

We next asked if MutS $\alpha$ might influence BLM helicase activity on a different DNA substrate. To that end, a forked DNA structure that mimics a simplified version of a replication fork was incubated with increasing amounts of BLM (8-25 fmol) and a fixed amount of MutS $\alpha$ complex (200 fmol). As shown in Figure 5B (chart), MutS $\alpha$ did not stimulate or inhibit BLM unwinding of a forked DNA structure.

\section{Discussion}

The molecular roles of the BLM gene product in the maintenance of genomic stability in human cells still remain to be defined. In the present study, we have shown that the Bloom's syndrome helicase interacts in vivo and in vitro with hMSH6, a protein involved in MMR. Hence, BLM makes physical interactions with components (hMSH6 and $\mathrm{hMLH}$ 1) of the two major MMR heterodimeric complexes, MutS $\alpha$ and MutL $\alpha$. We have demonstrated that BLM interacts directly with hMSH6 via two separate sites comprising the amino acids $340-770$ and $904-1417$ of BLM. In addition, we have shown that two regions of hMSH6 (amino acids 1-718 and 1000-1360) mediate the interaction with BLM. However, immunofluorescence data suggest that BLM and hMSH6 may only co-localise to a limited extent in response to DNA damage. Further work is required to identify whether co-localisation occurs more dramatically in cells exposed to stresses other than $\gamma$-irradiation.

Various experiments performed in the past three years have suggested a likely role for BLM in HR repair through its ability to disrupt synthetic D-loop substrates and/or to promote the ATP-dependent translocation of HJ (Karow et al., 2000; van Brabant et al., 2000; Mohaghegh et al., 2001; Yang et al., 2002), a function that may suppress inappropriate DNA recombination in vivo. Consistent with these observations, BLM has been shown to interact with RPA (Brosh et al., 2000), RAD51 (Wu et al., 2001), hMLH1 (Langland et al., 2001; Pedrazzi et al., 2001), and p53 (Wang et al., 2001), four proteins known also to influence HR repair. In the case of the BLM/p53 interaction, Yang et al. have demonstrated recently that purified recombinant p53 attenuates the ability of BLM to unwind synthetic HJs in vitro (Yang et al., 2002). Thus, a role for BLM as an 'anti-recombinase' in the suppression of genome instability is now suggested. Nevertheless, why should it be necessary for BLM to interact with mismatch repair proteins such as $\mathrm{hMSH} 6$ and $\mathrm{hMLH} 1$ during this or related HR processes? We suggest that the most likely answer to this question lies in the property of at least some MMR proteins to participate in HR in addition to their role in the post-replicative mismatch repair (reviewed in Bella- cosa, 2001). MMR proteins have been shown to be antirecombinogenic in yeast and bacteria, to be involved in regulation of heteroduplex length in yeast and mice, and to suppress homeologous recombination (reviewed in Modrich and Lahue, 1996; Evans and Alani, 2000; Harfe and Jinks-Robertson, 2000). In addition to these observations, the MutS $\alpha$ complex can bind with high affinity and specificity to HJ (Marsischky et al., 1999), and thus may be involved in the HR repair process in vivo by rendering the HJ more accessible to other processing components. These data, combined with evidence that BLM serves to prevent inappropriate HR during DNA replication, lead us to propose that MMR proteins such as $\mathrm{hMSH} 6$ and $\mathrm{hMLH} 1$ may assist BLM to perform its 'antirecombinase' function by modulating its branch migration activity leading to the restoration of a functional replication fork structure. In this way, MMR proteins could serve as 'docking sites' to position BLM at sites of HR repair. However, we could not observe any effect of the purified recombinant MutS $\alpha$ complex on the ability of BLM to unwind synthetic $\mathrm{HJ}$ in our in vitro helicase experiments. Nevertheless, it seems unlikely that BLM and MutS $\alpha$ act alone and therefore any modulation of BLM activity, if it occurs, may require one or more additional components of the MMR or HR machinery.

Clearly, the physical interactions that exist between BLM and hMSH6, and between BLM and hMLH1 (Langland et al., 2001; Pedrazzi et al., 2001), strongly suggest that BLM is intimately linked to some aspect(s) of the MMR process. Given that Bloom's syndrome cells are MMR proficient, BLM is unlikely to function in 'generalised' MMR, but is more likely involved in the regulation of genetic recombination. Further establishing these links by more sophisticated functional assays is a critical next step in the investigation of the functional connections between BLM and MMR proteins during HR. It will also be interesting to determine whether WRN and RECQL4, other members of the RecQ family helicases that are defective in Werner's and Rothmund-Thomson syndromes, respectively, also interact physically and functionally with the MMR proteins. These studies will certainly lead to a deeper understanding of the fundamental roles of the MMR proteins and RecQ helicases in DNA replication and $H R$ repair.

\section{Materials and Methods}

\section{Hela Nuclear Extracts}

Extracts were prepared as described in Perkins et al. (1994).

\section{Construction of Plasmids}

The different $\mathrm{hMSH} 6$ plasmids for in vitro transcription were generated by PCR using the $h M S H 6$ cDNA as a template, followed by cloning into vectors of the pCite- 4 series (Novagen, Madison, USA). For constructs used in interaction site mapping on BLM, see Pedrazzi et al. (2001). Sequences of all plasmids and construction schemes are available upon request. 


\section{Co-Immunoprecipitation Experiments}

Two-hundred $\mu \mathrm{g}$ of Hela cell nuclear extracts were incubated

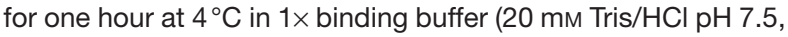
$5 \mathrm{~mm} \mathrm{MgCl}, 1 \mathrm{~mm}$ PMSF, $1 \mathrm{~mm}$ glutathione, $0.1 \mathrm{~mm}$ dNTPs, $50 \mu \mathrm{g} / \mathrm{ml} \mathrm{BSA}$ ), supplemented with $10 \%$ sucrose and $1 \times$ protease inhibitor cocktail, EDTA-free (Roche, Basel, Switzerland) and $0.05 \mu \mathrm{g} / \mathrm{ml}$ polydldC (Fluka, Buchs, Switzerland) with an effective salt concentration of $80 \mathrm{~mm} \mathrm{NaCl}$. Two $\mu \mathrm{g}$ of the mouse monoclonal anti-hMSH6 antibody (MCA 1687; Serotec, Oxford, UK) or a mouse IgG control antibody for the hMSH6 were used in the immunoprecipitation experiments. Alternatively, $4 \mu \mathrm{g}$ of the polyclonal goat anti-BLM antibody C-18 (Santa Cruz, Santa Cruz, USA) or a goat IgG control antibody were used in BLM immunoprecipitations. In each case, the incubations were continued for two hours. Twenty $\mu$ l of protein G Dynabeads (Dynal, Hamburg, Germany) were added to the solution and the incubation was continued for a further $1.5 \mathrm{~h}$ before the matrix-bead proteins were isolated according to the instructions of the manufacturer. The beads were washed five times with $200 \mu \mathrm{l}$ of binding buffer $(100 \mathrm{mM} \mathrm{NaCl}$ for IP with antihMSH6 antibody, and $100 \mathrm{~mm} \mathrm{NaCl}$ plus $150 \mathrm{~mm} \mathrm{KCl}$ for antiBLM antibody) before elution with $2 \times$ Laemmli buffer. The immunoprecipitated proteins were subjected to Western blot analysis using a polyclonal anti-hMSH6 antibody (Palombo et al., 1995) or the polyclonal anti-BLM IHIC33 antibody (Wu et al., 2000b). Detection was performed using ECL (Amersham Pharmacia Biotech, Uppsala, Sweden) following the manufacturer's instructions.

\section{Far-Western Analysis}

This assay was performed essentially as described previously (Wu et al., 2000b). Briefly, $1 \mu \mathrm{g}$ of purified hMutS $\alpha, 1 \mu \mathrm{g}$ of BSA and $0.5 \mu \mathrm{g}$ of purified hMutL $\alpha$ were subjected to SDS-PAGE and transferred to nitrocellulose filters. After renaturation and blocking, the filters were incubated for $60 \mathrm{~min}$ in a solution containing BLM $(1 \mu \mathrm{g} / \mathrm{ml})$ in TBS supplemented with $0.25 \%$ milk, $0.3 \%$ Tween 20, $1 \mathrm{~mm}$ DTT and $1 \mathrm{~mm}$ PMSF. After extensive washing, conventional Western blotting was performed to detect the presence of BLM (antibody IHIC33). The inputs were visualised with antibodies against hMLH1 (G168-15; Pharmingen, San Diego, USA), hMSH2 (Ab-2; Calbiochem, San Diego, USA), hMSH6 (21F10, Serotec) and BLM (IHIC33). For Figure 1A, MutS $\alpha$, MutL $\alpha$ and protein D were directly spotted onto the membrane in increasing amounts $(0,0.4,0.8,1.6,3.2$ and 6.4 pmoles $)$ and incubated with BLM $(3 \mu \mathrm{g} / \mathrm{ml})$ as described below for the in vitro binding assay. Detection of bound BLM protein was as outlined above.

\section{In Vitro Binding Assay}

Different amounts of recombinant BLM and BSA were directly spotted onto a nitrocellulose membrane (Micron Separation Inc., Westborough, USA), while hMutS $\alpha$ was subjected to SDS-PAGE prior to transfer to the nitrocellulose membrane followed by renaturation/denaturation steps as described for the Far Western assay. After blocking for one hour at room temperature using TBS supplemented with $5 \%$ non-fat milk and $0.5 \%$ Tween, the membrane was incubated for three hours at $4^{\circ} \mathrm{C}$ with different proteins that were [35S]-labelled using the TNT T7 quick-coupled transcription/translation system (Promega, Mannheim, Germany) in $1 \mathrm{ml}$ TBS with $0.5 \%$ Tween and $0.1 \%$ BSA. Fifty $\mu \mathrm{l}$ (of BLM fragments and full-length hMSH6-hMSH2 complex) or $75 \mu \mathrm{l}$ (hMSH6 fragments) of the in vitro transcription and translation reactions were used for each incubation. After extensive washing with TBS supplemented with $0.5 \%$ Tween, the mem- branes were dried and exposed to a Phosphorlmager (Molecular Dynamics, Sunnyvale, USA).

\section{Indirect Immunofluorescence Analysis}

Indirect Immunofluorescence Analysis was performed essentially as described in Wu et al. (2000b), with slight modifications. Cells were grown on coverslips and fixed with $4 \%$ paraformaldehyde, $250 \mathrm{~mm}$ HEPES, pH 7.4 at $4{ }^{\circ} \mathrm{C}$ for $20 \mathrm{~min}$, and then permeabilised in $0.1 \%$ Triton X-100 in PBSA for 20 min. After washing 5 times in PBSA for 20 min, blocking was carried out at $37^{\circ} \mathrm{C}$ for $20 \mathrm{~min}$ in $10 \%$ foetal bovine serum, $0.1 \%$ Triton $\mathrm{X}-100$ in PBSA. The coverslips were incubated for $1 \mathrm{~h}$ at $37^{\circ} \mathrm{C}$ with the primary antibodies IHIC33, or hMSH6/GTBP (BD Transduction Laboratories, Lexington, USA), which were diluted in the above blocking solution at 1:200, and 1:800, respectively. Five washes in PBSA for 20 min were followed by incubation with anti-mouse Cy3 (Sigma, St. Louis, USA), anti-rabbit fluorescein isothiocyanate (Dako, Glostrup, Denmark) or anti-rabbit AlexaFluor 488 (Molecular Probes, Eugene, USA) secondary antibodies for 1 hour at $37^{\circ} \mathrm{C}$ at $1 / 800,1 / 200$ and $1 / 800$ dilutions, respectively. Cells were washed five times in PBSA, and the DNA was stained using Hoechst 33258 at $50 \mathrm{ng} / \mathrm{ml}$. Stained slides were mounted in $90 \%$ glycerol, $20 \mathrm{~mm}$ Tris- $\mathrm{HCl}, \mathrm{pH} 8.0$, and $50 \mu \mathrm{g} / \mathrm{ml}$ paraphenylenediamine. Slides were viewed at $100 \times$ magnification on a Zeiss Axioskop microscope. Image acquisition and analysis were performed using the AxioVision (Zeiss, Jena, Germany) software, and the images were merged using Adobe Photoshop.

\section{DNA Helicase Assays}

Approximately $0.075 \mathrm{fmol}$ of oligonucleotide-based 4-way junction or forked duplex substrate (Mohaghegh et al., 2001) were incubated with the indicated amounts of BLM and hMutS $\alpha$ proteins in a $10 \mu \mathrm{l}$ reaction volume at $37^{\circ} \mathrm{C}$ for 45 minutes in helicase buffer (33 mM Tris-acetate $\mathrm{pH} 7.8,1 \mathrm{~mm}$ Mg-acetate, $66 \mathrm{~mm}$ K-acetate, $0.1 \mathrm{mg} / \mathrm{ml} \mathrm{BSA}, 1 \mathrm{~mm}$ DTT, $1 \mathrm{~mm}$ ATP). The reaction was stopped by the addition of $1 / 10$ volume loading buffer (100 mM Tris-HCl, 100 mm EDTA, bromophenol blue and xylene cyanol). The samples were electrophoresed on $10 \%$ acrylamide gels in $1 \times \mathrm{TBE}$ at $25 \mathrm{~mA}$ for $1 \mathrm{~h}$ at $4^{\circ} \mathrm{C}$. The gels were dried at $80^{\circ} \mathrm{C}$ for $30 \mathrm{~min}$ and subjected to autoradiography. Quantitative analysis of the rate of unwinding was performed on a Storm 840 Phosphorlmager and ImageQuant software (Molecular Dynamics).

\section{Acknowledgements}

We are grateful to Patrick Amstutz for the gift of purified phage protein D, to Pavel Janscak for purified MutS $\alpha$, and to Ulrich Hübscher for his support. This work was financed by grants from Bonizzi-Theler Foundation, EMDO Foundation, Gebert-Rüf Foundation, Walter Honegger Foundation, Stiftung für medizinische Forschung, Novartis Foundation, Olga Mayenfish Foundation, Sassella Foundation, Zürcher Krebsliga, EU Grant HPRN-CT-2002-00240, and Swiss National Science Foundation (no. 31-58798.99) to I.S. C.Z.B. and I.D.H. are supported by the Cancer Research UK and EU Grant HPRN-CT-2002 - 00240. C.Z.B. is a Marie Curie Fellow of the European Union (HPMF-CT$2000-00952)$. The generous support of the Swiss National Science Foundation (no. 31-68182.02) to J.J. is also gratefully acknowledged. 


\section{References}

Bellacosa, A. (2001). Functional interactions and signaling properties of mammalian DNA mismatch repair proteins. Cell Death Diff. 8, 1076-1092.

Bischof, O., Kim, S. H., Irving, J., Beresten, S., Ellis, N. A. and Campisi, J. (2001). Regulation and localization of the Bloom syndrome protein in response to DNA damage. J. Cell Biol. 153, 367-380.

Brosh, R. M., Jr., Li, J. L., Kenny, M. K., Karow, J. K., Cooper, M. P., Kureekattil, R. P., Hickson, I. D. and Bohr, V. A. (2000). Replication protein A physically interacts with the Bloom's syndrome protein and stimulates its helicase activity. J. Biol. Chem. 275, 23500-23508.

de Wind, N., Dekker, M., Berns, A., Radman, M. and te Riele, H. (1995). Inactivation of the mouse Msh2 gene results in mismatch repair deficiency, methylation tolerance, hyperrecombination, and predisposition to cancer. Cell 82, 321-330.

de Wind, N., Dekker, M., Claij, N., Jansen, L., van Klink, Y., Radman, M., Riggins, G., van der Valk, M., van't Wout, K. and te Riele, H. (1999). HNPCC-like cancer predisposition in mice through simultaneous loss of Msh3 and Msh6 mismatch-repair protein functions. Nature Genet. 23, 359-362.

Ellis, N. A., Groden, J., Ye, T. Z., Straughen, J., Lennon, D. J., Ciocci, S., Proytcheva, M. and German, J. (1995). The Bloom's syndrome gene product is homologous to RecQ helicases. Cell 83, 655-666.

Evans, E. and Alani, E. (2000). Roles for mismatch repair factors in regulating genetic recombination. Mol. Cell. Biol. 20, 78397844.

Gangloff, S., McDonald, J. P., Bendixen, C., Arthur, L. and Rothstein, R. (1994). The yeast type I topoisomerase Top3 interacts with Sgs1, a DNA helicase homolog: a potential eukaryotic reverse gyrase. Mol. Cell. Biol. 14, 8391-8398.

Garkavtsev, I. V., Kley, N., Grigorian, I. A. and Gudkov, A. V. (2001). The Bloom syndrome protein interacts and cooperates with p53 in regulation of transcription and cell growth control. Oncogene 20, 8276-8280.

German, J. (1993). Bloom syndrome: a mendelian prototype of somatic mutational disease. Medicine 72, 393-406.

German, J., Crippa, L. P. and Bloom, D. (1974). Bloom's syndrome. III. Analysis of the chromosome aberration characteristic of this disorder. Chromosoma 48, 361-366.

Harfe, B. D. and Jinks-Robertson, S. (2000). DNA mismatch repair and genetic instability. Annu. Rev. Genet. 34, 359-399.

laccarino, I., Marra, G., Palombo, F. and Jiricny, J. (1998). $\mathrm{hMSH} 2$ and hMSH6 play distinct roles in mismatch binding and contribute differently to the ATPase activity of hMutS $\alpha$. EMBO J. 17, 2677-2686.

Ishov, A. M., Sotnikov, A. G., Negorev, D., Vladimirova, O. V., Neff, N., Kamitani, T., Yeh, E. T., Strauss, J. F. and Maul, G. G. (1999). PML is critical for ND10 formation and recruits the PML-interacting protein daxx to this nuclear structure when modified by SUMO-1. J. Cell Biol. 147, 221-234.

Karow, J. K., Chakraverty, R. K. and Hickson, I. D. (1997). The Bloom's syndrome gene product is a 3'-5' DNA helicase. J. Biol. Chem. 272, 30611-30614.

Karow, J. K., Constantinou, A., Li, J. L., West, S. C. and Hickson, I. D. (2000). The Bloom's syndrome gene product promotes branch migration of Holliday junctions. Proc. Natl. Acad. Sci. USA 97, 6504-6508.

Kitao, S., Ohsugi, I., Ichikawa, K., Goto, M., Furuichi, Y. and Shimamoto, A. (1998). Cloning of two new human helicase genes of the RecQ family: biological significance of multiple species in higher eukaryotes. Genomics 54, 443-452.

Kitao, S., Shimamoto, A., Goto, M., Miller, R. W., Smithson, W.
A., Lindor, N. M. and Furuichi, Y. (1999). Mutations in RECQL4 cause a subset of cases of Rothmund-Thomson syndrome. Nature Genet. 22, 82-84.

Kleczkowska, H. E., Marra, G., Lettieri, T. and Jiricny, J. (2001). $\mathrm{hMSH} 3$ and hMSH6 interact with PCNA and colocalize with it to replication foci. Genes Dev. 15, 724-736.

Langland, G., Kordich, J., Creaney, J., Goss, K. H., LillardWetherell, K., Bebenek, K., Kunkel, T. A. and Groden, J. (2001). The Bloom's syndrome protein (BLM) interacts with MLH1 but is not required for DNA mismatch repair. J. Biol. Chem. 276, 30031-30035.

Luo, G., Santoro, I. M., McDaniel, L. D., Nishijima, I., Mills, M., Youssoufian, H., Vogel, H., Schultz, R. A. and Bradley, A. (2000). Cancer predisposition caused by elevated mitotic recombination in Bloom mice. Nature Genet. 26, 424-429.

Marsischky, G. T., Lee, S., Griffith, J. and Kolodner, R. D. (1999). Saccharomyces cerevisiae MSH2/6 complex interacts with Holliday junctions and facilitates their cleavage by phage resolution enzymes. J. Biol. Chem. 274, 7200-7206.

Meetei, A. R., Sechi, S., Wallisch, M., Yang, D., Young, M. K., Joenje, H., Hoatlin M. E. and Wang W. (2003). A multiprotein nuclear complex connects Fanconi anemia and Bloom syndrome. Mol. Cell. Biol. 23, 3417-3426.

Modrich, P. and Lahue, R. (1996). Mismatch repair in replication fidelity, genetic recombination, and cancer biology. Annu. Rev. Biochem. 65, 101-133.

Mohaghegh, P., Karow, J. K., Brosh Jr, R. M., Jr., Bohr, V. A. and Hickson, I. D. (2001). The Bloom's and Werner's syndrome proteins are DNA structure-specific helicases. Nucleic Acids Res. 29, 2843-2849.

Nakayama, K., Irino, N. and Nakayama, H. (1985). The recQ gene of Escherichia coli K12: molecular cloning and isolation of insertion mutants. Mol. Gen. Genet. 200, 266-271.

Palombo, F., Gallinari, P., laccarino, I., Lettieri, T., Hughes, M., D'Arrigo, A., Truong, O., Hsuan, J. J. and Jiricny, J. (1995). GTBP, a 160-kilodalton protein essential for mismatch-binding activity in human cells. Science 268, 1912-1914.

Pedrazzi, G., Perrera, C., Blaser, H., Kuster, P., Marra, G., Davies, S. L., Ryu, G. H., Freire, R., Hickson, I. D., Jiricny, J. and Stagljar, I. (2001). Direct association of Bloom's syndrome gene product with the human mismatch repair protein MLH1. Nucleic Acids Res. 29, 4378-4386.

Perkins, N. D., Agranoff, A. B., Duckett, C. S. and Nabel, G. J. (1994). Transcription factor AP-2 regulates human immunodeficiency virus type 1 gene expression. J. Virol. 68, 68206823.

Puranam, K. L. and Blackshear, P. J. (1994). Cloning and characterization of RECQL, a potential human homologue of the Escherichia coli DNA helicase RecQ. J. Biol. Chem. 269, 2983829845.

Raschle, M., Marra, G., Nystrom-Lahti, M., Schar, P. and Jiricny, J. (1999). Identification of hMutL $\beta$, a heterodimer of hMLH1 and hPMS1. J. Biol. Chem. 274, 32368-32375.

Sanz, M. M., Proytcheva, M., Ellis, N. A., Holloman, W. K. and German, J. (2000). BLM, the Bloom's syndrome protein, varies during the cell cycle in its amount, distribution, and co-localization with other nuclear proteins. Cytogenet. Cell Genet. 91, 217-223.

Sengupta, S., Linke, S. P., Pedeux, R., Yang, Q., Farnsworth, J., Garfield, S. H., Valerie, K., Shay, J. W., Ellis, N. A., Wasylyk, B. and Harris, C. C. (2003). BLM helicase-dependent transport of p53 to sites of stalled DNA replication forks modulates homologous recombination. EMBO J. 22, 1210-1222.

Stavropoulos, D. J., Bradshaw, P. S., Li, X., Pasic, I., Truong, K., Ikura, M., Ungrin, M. and Meyn, M. S. (2002). The Bloom syndrome helicase BLM interacts with TRF2 in ALT cells and pro- 
motes telomeric DNA synthesis. Hum. Mol. Genet. 11, 31353144.

Stewart, E., Chapman, C. R., Al-Khodairy, F., Carr, A. M. and Enoch, T. (1997). rqh1+, a fission yeast gene related to the Bloom's and Werner's syndrome genes, is required for reversible S phase arrest. EMBO J. 16, 2682-2692.

Sun, H., Karow, J. K., Hickson, I. D. and Maizels, N. (1998). The Bloom's syndrome helicase unwinds G4 DNA. J. Biol. Chem. 273, 27587-27592.

van Brabant, A. J., Ye, T., Sanz, M., German, I. J., Ellis, N. A. and Holloman, W. K. (2000). Binding and melting of D-loops by the Bloom syndrome helicase. Biochemistry 39, 14617-14625.

Wang, X. W., Tseng, A., Ellis, N. A., Spillare, E. A., Linke, S. P., Robles, A. I., Seker, H., Yang, Q., Hu, P., Beresten, S. et al. (2001). Functional interaction of p53 and BLM DNA helicase in apoptosis. J. Biol. Chem. 276, 32948-32955.

Wang, Y., Cortez, D., Yazdi, P., Neff, N., Elledge, S. J. and Qin, J. (2000). BASC, a super complex of BRCA1-associated proteins involved in the recognition and repair of aberrant DNA structures. Genes Dev. 14, 927-939.

Watt, P. M., Louis, E. J., Borts, R. H. and Hickson, I. D. (1995). Sgs1: a eukaryotic homolog of $E$. coli RecQ that interacts with topoisomerase II in vivo and is required for faithful chromosome segregation. Cell 81, 253-260.

Watt, P. M., Hickson, I. D., Borts, R. H. and Louis, E. J. (1996). SGS1, a homologue of the Bloom's and Werner's syndrome genes, is required for maintenance of genome stability in Saccharomyces cerevisiae. Genetics 144, 935-945.

Wu, L., Davies, S. L. and Hickson, I. D. (2000a). Roles of RecQ family helicases in the maintenance of genome stability. Cold Spring Harbor Symp. Quant. Biol. 65, 573-581.

Wu, L., Davies, S. L., North, P. S., Goulaouic, H., Riou, J. F., Turley, H., Gatter, K. C. and Hickson, I. D. (2000b). The Bloom's syndrome gene product interacts with topoisomerase III. J. Biol. Chem. 275, 9636-9644.

Wu, L., Davies, S. L., Levitt, N. C. and Hickson, I. D. (2001). Potential role for the BLM helicase in recombinational repair via a conserved interaction with RAD51. J. Biol. Chem. 276, 19375-19381.

Yamagata, K., Kato, J., Shimamoto, A., Goto, M., Furuichi, Y. and Ikeda, H. (1998). Bloom's and Werner's syndrome genes suppress hyperrecombination in yeast sgs 1 mutant: implication for genomic instability in human diseases. Proc. Natl. Acad. Sci. USA 95, 8733-8738.

Yang, Q., Zhang, R., Wang, X. W., Spillare, E. A., Linke, S. P., Subramanian, D., Griffith, J. D., Li, J. L., Hickson, I. D., Shen, J. C. et al. (2002). The processing of Holliday junctions by BLM and WRN helicases is regulated by p53. J. Biol. Chem. 277, 31980-31987.

Yankiwski, V., Marciniak, R. A., Guarente, L. and Neff, N. F. (2000). Nuclear structure in normal and Bloom syndrome cells. Proc. Natl. Acad. Sci. USA 97, 5214-5219.

Yu, C. E., Oshima, J., Fu, Y. H., Wijsman, E. M., Hisama, F., Alisch, R., Matthews, S., Nakura, J., Miki, T., Ouais, S., Martin, G. M., Mulligan, J. and Schellenberg, G. D. (1996). Positional cloning of the Werner's syndrome gene. Science 272, 258-262.

Received April 29, 2003; accepted June 23, 2003 